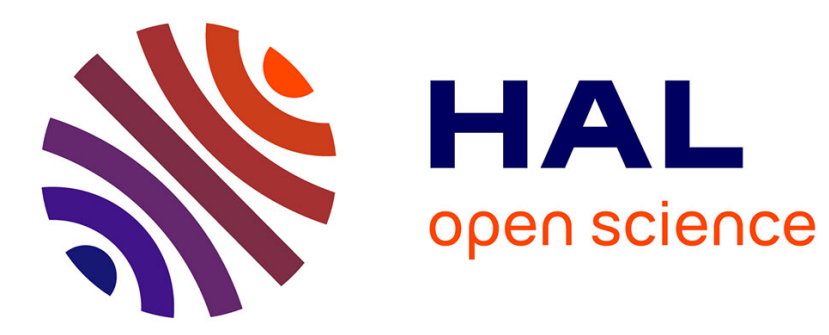

\title{
Optimal User Association in Multi-user MIMO Small Cell Networks
}

\author{
Sreenath Ramanath, Merouane Debbah, Eitan Altman
}

\section{To cite this version:}

Sreenath Ramanath, Merouane Debbah, Eitan Altman. Optimal User Association in Multi-user MIMO Small Cell Networks. 8th International Conference on Communication Systems and Networks (COMSNETS), Jan 2016, Bangalore, India. hal-01240266

\section{HAL Id: hal-01240266 https://inria.hal.science/hal-01240266}

Submitted on 8 Dec 2015

HAL is a multi-disciplinary open access archive for the deposit and dissemination of scientific research documents, whether they are published or not. The documents may come from teaching and research institutions in France or abroad, or from public or private research centers.
L'archive ouverte pluridisciplinaire HAL, est destinée au dépôt et à la diffusion de documents scientifiques de niveau recherche, publiés ou non, émanant des établissements d'enseignement et de recherche français ou étrangers, des laboratoires publics ou privés. 


\title{
Optimal User Association in Multi-user MIMO Small Cell Networks
}

\author{
Sreenath Ramanath ${ }^{1}$, Merouane Debbah ${ }^{2}$, Eitan Altman ${ }^{3}$ \\ ${ }^{1}$ Lekha Wireless Solutions, Bangalore, India; ${ }^{2}$ Huawei, Paris, France; ${ }^{3}$ INRIA, Sophia Antipolis, France;
}

\begin{abstract}
Dense Networks and large MIMO are two key enablers to achieve high data rates towards next generation 5G networks. In this context, we study user association in an interference limited Multi-user MIMO Small Cell Network. Extending on our previous findings, we derive explicit expressions for the optimal ratio of the number of antennas at the base station to the number of users that can associate to a base station in such a Network. This expressions are used to compute the actual number of users that can associate for a given interference level and other system parameters. Simulation results and numerical examples are provided to support our theoretical findings.
\end{abstract}

Keywords: Small cell networks; Multi-user MIMO; User association;

\section{INTRODUCTION}

Small cell networks (SCNs) and large (Massive) MIMO are envisaged as two of the important ingredients to satisfy mobile data demands of next generation 5G Networks [1], [2], [3], [4]. While, SCNs are dense deployment of short range (tens to few hundreds of meters) base stations which aim to improve capacity and coverage, massive MIMO aims to increase spectral efficiency (bits-per-hertz) and reduce interference by utilizing the spatial dimension. For effective network deployment, the base stations in the network need to be self organized, power efficient and portable. One of the needs of being self organized is being able to optimize the number of users that associate with a given base station under certain constraints like, power, interference level, etc. In this context, we study optimal user association in a multi-input multi-output (MIMO) small cell network, where multi-antenna base stations communicate with multiple single antenna users.

It is well known that dividing a macro cell into multiple small cells enhances coverage and capacity. Exploring the spatial dimension via multiple antenna at the transmitter $\left(N_{t}\right)$ and the receiver $\left(N_{r}\right)$ further enhances the capacity, which grows linearly as $\min \left(N_{t}, N_{r}\right)$.

Multi-user MIMO capacity has been studied in literature extensively (See for example [5], [6], [7], [8] and the references there in). Recent work in the context of multi-user MIMO, where multi-antenna base station, transmitting on $M$ antennas to $K$ single antenna users have been shown to achieve capacity gains which grow linearly as $\min (M, K)$, when channel state information is available [9]. Precoders [10], [11] are a popular choice that trades off complexity and performance to achieve this.

While the linear capacity growth holds for a single cell, the gains diminish in a multi cell network due to interference from neighboring base stations. Interference co-ordination and management techniques [12], [13], [14], [15] can mitigate these to a certain extent, albeit at the cost of additional circuitry and power constraints, which can be a significant overhead in the context of small cell networks. For example, if a small cell base station is battery operated, power consumption would significantly increase with additional circuitry. Even assuming the existence of power efficient interference co-ordination and management, during the course of operation, the small cell base stations might decide to shut off extra circuitry for efficient power utilization, depending on load, battery level, etc., It is in this context that we want to look at the user association problem; What is an optimal number of users that can associate with a small cell base station in such a network for a given level of interference?

Our approach to answer this question uses the sum-capacity expressions derived in the asymptotic regime using tools from Random Matrix Theory. In [16], the authors analyze capacity scaling in an interference limited multi-user MIMO small cell network, where the capacity expressions were derived as a function of number of base station antennas $M$, number of users $K$, both increasing asymptotically, while keeping the ratio $\beta=M / K$ a constant. Using these expressions, the problem of finding the optimal $\beta, \beta^{*}$ which maximizes the capacity gain was addressed and some simulation based examples were provided to compute $\beta^{*}$.

In this work, starting from the asymptotic capacity expressions, we specifically focus on the computation of $\beta^{*}$. Once $\beta^{*}$ is known, for any $M$ the number of antennas on the base station, the actual number of users $K^{*}$ can be derived.

Two methods are proposed for the computation of $\beta^{*}$; The first method uses an iterative zero finding algorithm to compute $\beta^{*}$, while the second one provides an explicit expression using the Lambert-W function. While the latter provides a quick means to compute $\beta^{*}$ using advanced mathematical tools to analyze the problem at hand, the former can be part of the software flow of a self organizing small cell base station, which is invoked whenever system resources (e.g., battery power) are to be efficiently managed.

User association has been studied extensively in literature (See [17], [18] and the reference there in) with different performance goals and various system parameters. While performance goals are to ensure certain QoS guarantees (throughput, coverage, delay, etc.), under instantaneous or average utilities, fairness constraints, load balancing, etc., system parameters address aspects like power budget, network deployment 


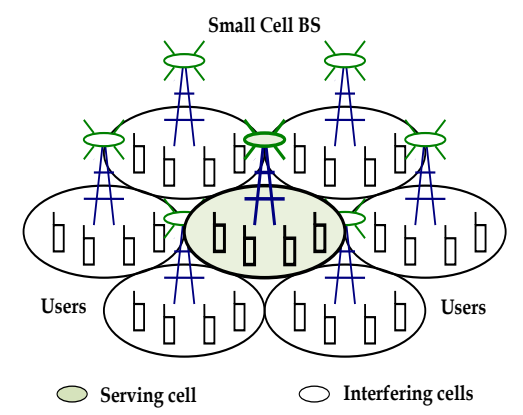

Fig. 1. Multi-user MIMO Small Cell Network: Base station with $M$ antennas, serving $K$ users.

(macro/micro/small cell, homogeneous/heterogeneous, regular/stochastic distribution of base stations/users), propagation medium (path-loss, fading/shadowing), interference (own cell and other cell), spatial configurations (SISO, MIMO), receiver structures (ZF, MMSE, ML), base station co-operation (centralized, distributed), etc. An analysis similar to our approach has been addressed in [19]. In this paper, the authors address user-base station association among other things in the context of large MIMO systems that employ precoding. Their system considers imperfect channel state information at the transmitter and per-user channel transmit correlation, in a single cell case. In contrast, we look at the problem in the downlink and are limited by multi cell interference. As the base station density increases, the system becomes interference limited.

The remainder of this paper is organized as follows: We introduce the multi cell system model in Section II. Section III revisits the capacity expressions for the single cell and multi cell case, derives the optimal $\beta, \beta^{*}$ and study optimal user association. Section IV provides simulation results and compares them against theoretical findings. Finally in Section $\mathrm{V}$, we provide conclusions.

Notations: In the following, boldface lower-case symbols represent vectors, capital boldface characters denote matrices ( $\mathbf{I}_{N}$ is the $N \times N$ identity matrix). The Hermitian transpose is denoted $(\cdot)^{\mathrm{H}}$. The operator $\operatorname{tr}[\mathbf{X}]$ represents the trace of matrix $\mathbf{X}$. All logarithms are base-2 logarithms.

\section{System Model AND Assumptions}

We discuss the system model in this section. For simplicity and to be able to keep the analysis tractable, we consider a multi cell Wyner-type model [20], for example as shown in fig. (1). The cell at the center is our reference. The users in this cell experience interference from the neighboring base stations. Each cell serves $K$ users from a base station with $M$ antennas. We assume that the base station antennas are uncorrelated. The information from the base station to its user set is precoded assuming channel state information (CSI) is available. i.e., each base station knows perfectly the channel towards the users in its cell, but not the interfering channels. Users receive desired signal plus interference from adjacent base stations. We consider Zero Force precoding (also known as Channel Inversion precoding) at the transmitter. The transmitted signals from the base stations undergo path-loss and Rayleigh fading. We assume that the channel is constant for some interval long enough for the transmitter to learn and use it until it changes to a new value. Further, all users are assumed to have the same average (but not instantaneous) received signal power, so our model assumes that the users are at similar distances ${ }^{1}$ from the base station and are not in deep shadow fades.

\section{Single Cell AND Multi Cell Asymptotic CAPACITY}

In this Section, we recall the asymptotic results derived for capacity in the single cell and multi cell case ([16]).

\section{A. Single cell}

Without loss of generality, we consider cell 0 . The signal received by users in this cell is

$$
\mathbf{y}=\mathbf{H x}+\mathbf{n} .
$$

where, $\mathbf{H}$ is the $K \times M$ channel matrix with zero-mean unit-variance i.i.d complex Gaussian entries, $\mathbf{x}=\mathbf{G s}$ is the transmit vector obtained by linear precoding of the symbol vector $\mathbf{s}$ with the precoding matrix $\mathbf{G}$. Symbol $s_{k} \in \mathbf{s}$ for any user $k$ is complex Gaussian with zero mean and unit variance. The $M \times K$ linear precoding matrix is defined as

$$
\mathbf{G}=\alpha \mathbf{H}^{\mathrm{H}}\left(\mathbf{H H}^{\mathrm{H}}\right)^{-1} \text {. }
$$

where $\alpha$ is chosen appropriately to satisfy the total transmit power constraint $\operatorname{tr}\left(\mathrm{E}\left[\mathbf{x x}^{H}\right]\right) \leq \operatorname{tr}\left(\mathbf{G G}^{H}\right) \leq P$.

Now the received vector in Cell 0 is

$$
\mathbf{y}=\alpha \mathbf{s}+\mathbf{n} .
$$

The parameter $\alpha$ which satisfies the transmit power constraint and depends only on the channel realization $\mathbf{H}$ is given by

$$
\alpha^{2}=\frac{P}{\operatorname{tr}\left(\left(\mathbf{H H}^{\mathrm{H}}\right)^{-1}\right)}
$$

The SNR (signal to noise ratio) for any user $k$ is defined as

$$
\eta_{k}=\frac{\mathrm{E}_{s}\left[\left|\alpha s_{k}\right|^{2}\right]}{\mathrm{E}\left|n_{k}\right|^{2}}=\frac{\alpha^{2}}{\sigma^{2}} .
$$

is independent of the selected user. $\sigma^{2}$ is the noise variance.

The ergodic capacity for user $k$ is

$$
C_{k}=\log \left(1+\eta_{k}\right) \text {. }
$$

and the sum-rate is

$$
\mathcal{R}_{\mathrm{ZF}}=\sum_{k=1}^{K} \log \left(1+\eta_{k}\right) .
$$

\footnotetext{
${ }^{1}$ Example, users in a cluster or users belonging to a class who satisfy a certain average SNR constraint
} 
1) Asymptotic analysis for a Single cell: $\alpha$ is a function of $\mathbf{H}$ and as $M, K \rightarrow \infty, \alpha$ tends to a constant. Thus the sum-rate can be written as

$$
\mathcal{R}_{\mathrm{ZF}}=K \log \left(1+\eta_{k}\right)
$$

Let us denote $M / K=\beta$. In the asymptotic limits, as $M, K \rightarrow \infty$, with $\beta=M / K$ a constant and $\rho=P / \sigma^{2}$, the sum rate is derived [16] as

$$
\mathcal{R}_{\mathrm{ZF}}=K \log (1+\rho(\beta-1)) \text { for } \beta \geq 1
$$

The rate-per-antenna is given by

$$
\frac{\mathcal{R}_{\mathrm{ZF}}}{M}=\frac{1}{\beta} \log (1+\rho(\beta-1)) .
$$

As $\beta \rightarrow 1, \mathcal{R}_{\mathrm{ZF}} / M \rightarrow 0$, which implies that the sum rate of channel inversion does not increase linearly with $M$ (or $K$ )

2) Optimizing $\beta$ for the Single cell: Now we look for a value $\beta^{\star}$ of the ratio $M / K$ such that, for a fixed number of transmit antennas $M$, the sum-rate $\mathcal{R}_{\mathrm{ZF}}(\beta)$ is maximized. By differentiating eqn. (10) with respect to $\beta$ and setting the derivative to zero, $\beta^{*}$ is the solution of the fixed point equation

$$
\rho \beta^{*}=\left(1+\rho\left(\beta^{*}-1\right)\right) \log \left(1+\rho\left(\beta^{*}-1\right)\right)
$$

To find $\beta^{*}$, we can use numerical methods like the zero finding algorithm. Let

$$
\mathbf{g}\left(\beta_{l}\right)=\frac{1}{\rho}\left(1+\rho\left(\beta_{l}-1\right)\right) \log \left(1+\rho\left(\beta_{l}-1\right)\right)
$$

Now, we use the following steps to find $\beta^{*}$.

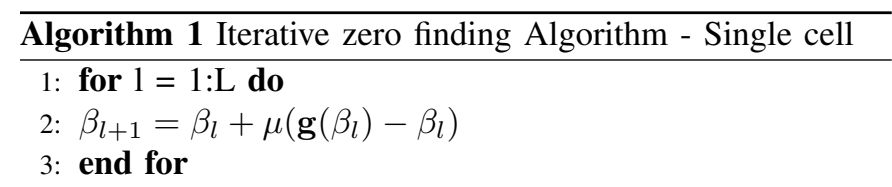

For large $L, \beta_{L} \rightarrow \beta^{*}$

The solution for the fixed point equation can also be found explicitly using the Lambert W-function ${ }^{2}$ as

$$
\beta^{*}=\frac{(\rho-1)(1+\zeta)}{\rho \zeta}
$$

$$
\text { where, } \zeta=\text { Lambert } W\left[(\rho-1) e^{-1}\right]
$$

Next we proceed to find the number of users that can associate with a base station. We fix $M$, the number of antennas on the BS and compute the asymptotic number of users as $K^{*}=K_{\text {asymptotic }}=M / \beta^{*}$. The actual number of users that can associate is always an integer and is given by

$$
K_{\text {actual }}=\left\lfloor K^{*}\right\rfloor .
$$

\section{B. Multi cell}

In this section, we study the effect of multi cell interference. Without loss of generality, we consider users in Cell 0 affected

\footnotetext{
${ }^{2}$ If $W(x)$ is a Lambert W-function, it satisfies $x=W(x) e^{W(x)}$ for any $x \in \mathbb{C}$.
}

by interference from adjacent base-stations as shown in fig. 1 . Cell $C_{0}$ is at the center. Neighbors are designated Cell $C_{1}, C_{2}$ ... $C_{N}$.

Following our analysis of the single cell case, the received vector for users of cell $C_{0}$, is

$$
\begin{aligned}
\mathbf{y} & =\mathbf{H}_{0} \mathbf{G}_{0} \mathbf{s}_{0}+\sqrt{\gamma_{1}} \mathbf{H}_{01} \mathbf{G}_{1} \mathbf{s}_{1}+\sqrt{\gamma_{1}} \mathbf{H}_{01} \mathbf{G}_{1} \mathbf{s}_{2} \\
& +\ldots+\sqrt{\gamma_{N}} \mathbf{H}_{0 N} \mathbf{G}_{N} \mathbf{s}_{N}+\mathbf{n} .
\end{aligned}
$$

As before, $\mathbf{H}_{0}$ is the channel matrix from base station in cell $\mathbb{C}_{0}$ to its users. $\mathbf{H}_{01} \ldots \mathbf{H}_{0 N}$ are interfering channels from cell $\mathbb{C}_{1} \ldots \mathbb{C}_{N}$, respectively. $\mathbf{G}_{1} \ldots \mathbf{G}_{N}$ are precoding matrices for users in cell $\mathbb{C}_{1} \ldots \mathbb{C}_{N}$, respectively. $\gamma_{1} \ldots \gamma_{N}$ are signal (interference) attenuation.

As stated earlier, all users in cell $\mathbb{C}_{0}$ are assumed to experience similar average received signal plus interference power. Thus $\gamma_{1}=\gamma_{2}=\ldots=\gamma_{N}=\gamma$.

The precoding matrices in cell $i$ can be written as

$$
\mathbf{G}_{i}=\alpha_{i} \mathbf{H}_{i}^{\mathrm{H}}\left(\mathbf{H}_{i} \mathbf{H}_{i}^{\mathrm{H}}\right)^{-1}
$$

The ergodic capacity for user $k$ in cell $\mathbb{C}_{0}$ is expressed as

$$
C_{k}=\log \left(1+\frac{\alpha_{0}^{2}}{\mathrm{E}\left[\left|n_{k}\right|^{2}\right]}\right)
$$

Where, $n_{k}$ is the $k^{t h}$ element of the covariance matrix $\mathbf{n}$. The expectation of this matrix can be written as

$$
\begin{aligned}
\mathrm{E}\left[\mathbf{n n}^{\mathrm{H}}\right] & =\gamma \mathbf{H}_{01} \mathbf{G}_{1} \mathbf{G}_{1}^{\mathrm{H}} \mathbf{H}_{01}^{\mathrm{H}}+\ldots \\
& +\gamma \mathbf{H}_{0 N} \mathbf{G}_{N} \mathbf{G}_{N}^{\mathrm{H}} \mathbf{H}_{0 N}^{\mathrm{H}}+\sigma^{2} \mathbf{I}
\end{aligned}
$$

Since, $\mathrm{E}\left[\left|n_{1}\right|^{2}\right]=\mathrm{E}\left[\left|n_{2}\right|^{2}\right] \ldots=\mathrm{E}\left[\left|n_{k}\right|^{2}\right]$

$$
\text { We can write, } \begin{aligned}
\mathrm{E}\left[\left|n_{k}\right|^{2}\right] & \rightarrow \frac{1}{K} \sum_{k=1}^{K} \mathrm{E}\left[\left|n_{i}\right|^{2}\right] \\
& =\frac{1}{K} \operatorname{tr}\left(\mathrm{E}\left[\mathbf{n} \mathbf{n}^{\mathrm{H}}\right]\right)
\end{aligned}
$$

1) Asymptotic analysis for the Multi cell: As $K, M \rightarrow \infty$, It can be shown that the expectation in the expression above reduces ([16] to,

$$
\mathrm{E}\left[\left|n_{k}\right|^{2}\right] \rightarrow \alpha_{1}^{2} \gamma \frac{1}{\beta-1}+\ldots+\alpha_{N}^{2} \gamma \frac{1}{\beta-1}+\sigma^{2}
$$

And hence, the sum-rate is

$$
\mathcal{R}_{\mathrm{ZF}}=K \log \left(1+\frac{\alpha_{0}^{2}(\beta-1)}{\alpha_{1}^{2} \gamma+\ldots+\alpha_{N}^{2} \gamma+\sigma^{2}(\beta-1)}\right)
$$

For large $(K, M)$,

$$
\begin{gathered}
\frac{\alpha_{0}^{2}}{\sigma^{2}}=\frac{\alpha_{1}^{2}}{\sigma^{2}}=\ldots=\frac{\alpha_{N}^{2}}{\sigma^{2}} \rightarrow \rho(\beta-1), \text { where } \rho=P / \sigma^{2} \\
\text { Thus, } \mathcal{R}_{\mathrm{ZF}}=K \log \left(1+\frac{\rho(\beta-1)}{1+N \gamma \rho}\right)
\end{gathered}
$$




$$
\text { Re-writing, } \frac{\mathcal{R}_{\mathrm{ZF}}}{M}=\frac{1}{\beta} \log \left(1+\frac{\rho(\beta-1)}{1+N \gamma \rho}\right)
$$

We observe that when $\gamma=0$ (when there is no interference), the capacity formula is that of the single cell case.

As $\beta \rightarrow 1, \mathcal{R}_{\mathrm{ZF}} / M \rightarrow 0$, which implies that the sum rate of channel inversion does not increase linearly with $M$ (or $K$ )

2) Optimizing $\beta$ for the Multi cell: Following on similar lines of the single cell case, we now look for a value $\beta^{\star}$ of the ratio $M / K$ such that, for a fixed number of transmit antennas $M$, the sum-rate $\mathcal{R}_{\mathrm{ZF}}(\beta)$ is maximized. By differentiating eqn. (24) with respect to $\beta$ and setting the derivative to zero, $\beta^{*}$ is the solution of the equation

$$
\rho \beta^{*}=\left[\rho\left(\beta^{*}-1\right)+(1+N \gamma \rho)\right] \log \left[1+\frac{\rho\left(\beta^{*}-1\right)}{1+N \gamma \rho}\right]
$$

One can observe that by setting $\gamma=0$, we fall back to the equation (11) of the single cell case.

To solve for $\beta^{*}$ iteratively, as in the single cell case, Let

$f\left(\beta_{l}\right)=\frac{1}{\rho}\left[\rho\left(\beta_{l}-1\right)+(1+N \gamma \rho)\right] \log \left[1+\frac{\rho\left(\beta_{l}-1\right)}{1+N \gamma \rho}\right]$

As before, we use the following steps to find $\beta^{*}$.

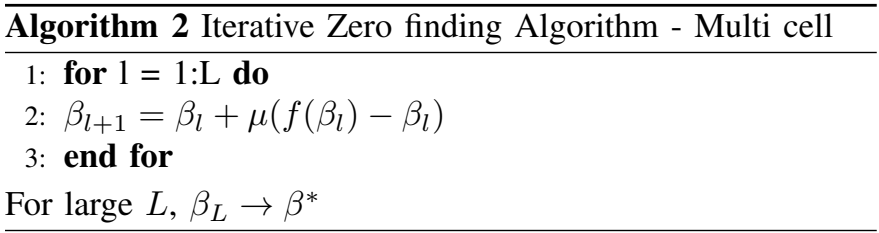

As before, the solution for the fixed point equation can also be found explicitly as a function of the Lambert $\mathrm{W}$-function

$$
\begin{aligned}
\beta^{*} & =\frac{(\rho-1)(1+\zeta)(1+N \gamma)}{\rho \zeta} \\
\text { where, } \zeta & =\text { Lambert } W\left(\frac{\rho-(1+N \gamma)}{e^{(1+N \gamma)}}\right)
\end{aligned}
$$

As discussed in the single cell case, the optimal number of users in the asymptotic limit is given by $K^{*}=K_{\text {asymptotic }}=$ $M / \beta^{*}$, from which the actual number of users that can associate will be $K_{\text {actual }}=\left\lfloor K^{*}\right\rfloor$.

\section{Simulation RESUlts}

Following the analysis from previous sections, we recall that $\beta^{*}$ is the ratio $M / K$, which maximizes the sum-rate. Thus if we fix $M$, the number of antennas on the BS, we can compute the optimal number of users that can associate in the asymptotic limit as $K^{*}=K_{\text {asymptotic }}=M / \beta^{*}$. Now the actual number of users that can associate is always an integer given by $K_{\text {actual }}=\left\lfloor K^{*}\right\rfloor$. For a fixed number of BS antennas $M, \beta^{*}$ and hence $K^{*}$ will be a function of the average SNR experienced by the user $\rho$ and the interference level $\gamma$. We are interested to study how the actual number of users that can associate with the BS vary over a range of SNR and $\gamma$ for the multi cell model (fig. 1) presented in Section II.

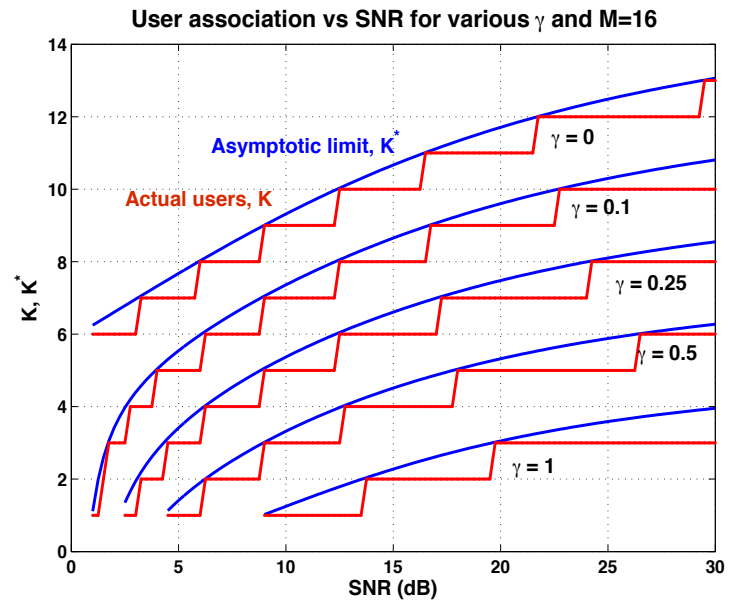

Fig. 2. User association for $M-16$ transmit antennas, interference level $\gamma \in(0,1)$ as a function of average SNR experienced by the user.

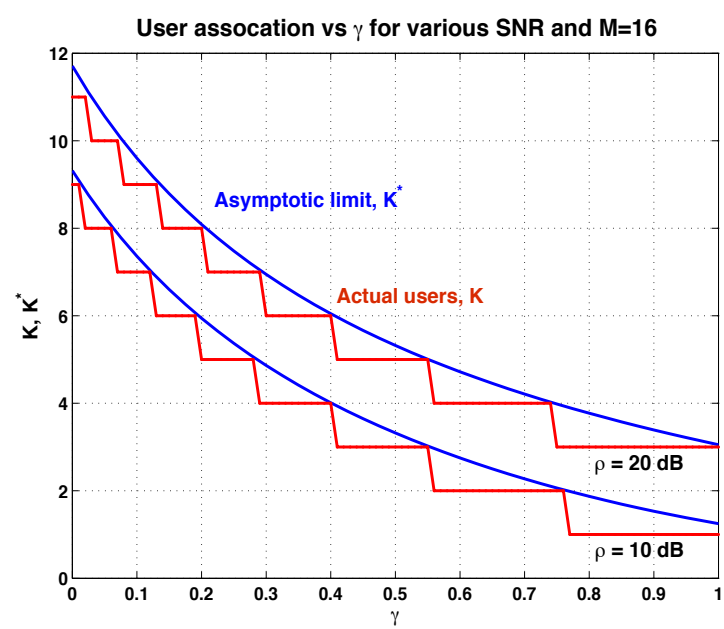

Fig. 3. User association for $M-16$ transmit antennas, SNR $\rho=10 d B, 20 d B$ as a function of Interference level $\gamma \in(0,1)$.

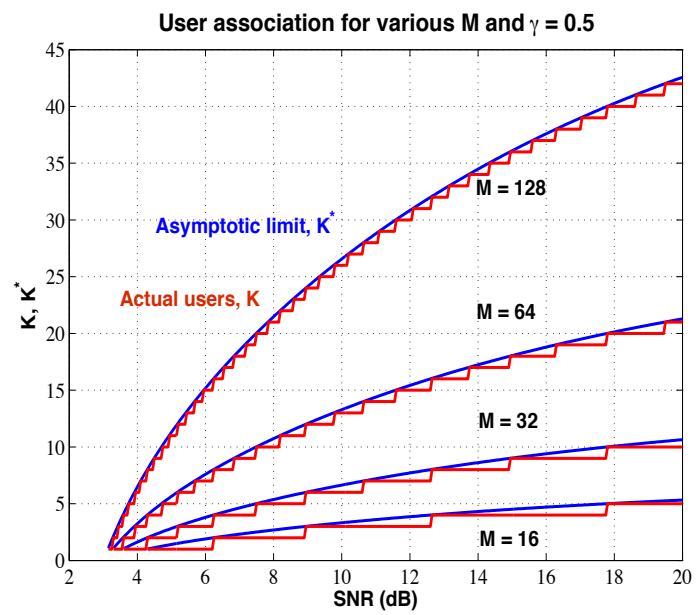

Fig. 4. User association as the number of transmit antennas $M$ scales up, Interference level $\gamma=0.5$ 
In fig. 2, we plot user association as a function of SNR for various interference levels. We observe that over a given range of SNR, the actual number of users associated do not change. This implies that the base station can benefit by transmitting at a power which is close to the lower limit for a certain actual number of associated users. For example, with an interference level corresponding to $\gamma=0.25$, we observe (See fig. 2) that the base station can associate with 7 users over a range of SNRs from 17 to $24 \mathrm{~dB}$. If the BS wants to associate with more users, it has to compensate by transmitting at a higher power level where-in the average SNR experienced by the user will be greater than $24 \mathrm{~dB}$.

In a similar setting, we fix the average SNR experienced by the users and study user association over a range of interference levels $\gamma \in(0,1)$ (see fig. 3). We observe that the number of actual users that can associate remains constant over a range of $\gamma$. For example, with an average SNR constraint of $20 \mathrm{~dB}$, the number of users that can associate with the BS remains at 6 for the range of interference $\gamma \in(0.3,0.4)$. As the base station density increases, the interference level limits the number of users that can associate.

Next we study user association as the number of antennas at the BS scales up. $\gamma$ is fixed to $0.5, M \in(16,32,64,128)$. In fig. 4, we plot this as a function of average SNR. As observed previously, the actual number of users that associate with the BS remains fixed over a range of SNR. But the range itself is narrower as the number of BS antennas scales up. This is expected as $K_{\text {actual }}$ will start approaching the asymptotic limit $K_{\text {asymptotic }}$ as the number of antennas at the BS grow large. For example (See fig. 4), with an interference level set to $\gamma=0.5$, we can observe that the number of users that associate, with the average SNR set at $16 \mathrm{~dB}$ is 4 for $M=16$ and increases to 18 when $M=64$. Note that the number of users remain at 4 over the range of SNRs from 12.5-17.5 $\mathrm{dB}$ for $M=16$, while the range spans $15-16.5 \mathrm{~dB}$ for 18 users when $M=64$. Thus the range of SNRs is narrower as $M$, the number of antennas at the BS scales up. Further, the rate per user remains almost constant as $M$ increases as the system operates with an overall power constraint $\mathrm{P}$, but, with an objective of maximizing the sum rate. For the example considered above this is found to be $1.406 \mathrm{bits} / \mathrm{sec} / \mathrm{Hz}$ per user for $M=16$ and $1.531 \mathrm{bits} / \mathrm{sec} / \mathrm{Hz}$ per user for $M=64$ when the average SNR is about $20 \mathrm{~dB}$. The asymptotic rates for both the cases was $1.579 \mathrm{bits} / \mathrm{sec} / \mathrm{Hz}$ per user.

\section{COnclusions}

Small cell networks in conjunction with Massive MIMO are gearing up to deliver high data rates envisaged for next generation wireless networks. In this context, we studied the problem of user association in interference limited multiuser MIMO small cell networks. Starting from ergodic sum capacity derived in an asymptotic setting, we derived explicit expressions for the optimal ratio of the number of antennas at the base station to the number of users that can associate with the base station. From these expressions, we derive the actual number of users that can associate with a base station for the system under consideration. Simulation results and numerical examples support our theoretical findings, even when the number of antennas on the base station and users are finite and fits well into the current MIMO and futuristic large MIMO deployments.

\section{ACKNOWLEDGMENT}

This work was partly supported by the Indo-French Centre for Applied Mathematics (IFCAM).

\section{REFERENCES}

[1] J. Hoydis, M. Kobayashi and M. Debbah, "Green small-cell networks", IEEE Vehicular Technology Magazine, 6 (1), 37-43.

[2] J. G. Andrews, H. Claussen, M. Dohler, S. Rangan and M. C. Reed, "Femtocells: Past, present, and future", IEEE Journal on Selected Areas in Communications, Vol 30, Apr 2012.

[3] K. Hosseini, J. Hoydis, S. Ten Brink and M. Debbah, Massive MIMO and small cells: How to densify heterogeneous networks, Proceedings of ICC 2013.

[4] E. G. Larsson, F. Tufvesson, O. Edfors, and T. L. Marzetta, "Massive MIMO for next generation wireless systems," IEEE Communications Magazine, Vol. 52, Feb 2014.

[5] H. Viswanathan and S. Venkatesan, "Asymptotics Of Sum Rate for Dirty Paper Coding and Beamforming In Multiple-Antenna Broadcast Channels", Proceedings of Allerton 1998.

[6] B. Hochwald and S. Vishwanath, "Space-Time Multiple Access: Linear Growth in the Sum Rate", Proceedings of Allerton 2002.

[7] A. Lozano, A. M. Tulino and S. Verdu, "Multiple-antenna capacity in the low-power regime", IEEE Trans. on Information Theory, Vol. 49, 2003.

[8] H. Weingarten, Y. Steinberg and S. Shamai, "The Capacity Region of the Gaussian Multiple-Input Multiple-Output Broadcast Channel", IEEE Trans. on Information Theory, Vol. 52, 2006.

[9] N. Jindal and A. Goldsmith, "Dirty-paper coding versus TDMA for MIMO Broadcast channels", IEEE Trans. on Information Theory, Vol. 51, 2005.

[10] C. Peel, B. Hochwald, and A. Swindlehurst, "A Vector-Perturbation Technique for Near-Capacity Multiantenna Multiuser Communication Part I: Channel Inversion and Regularization”, IEEE Trans. on Communications, Vol. 53, 2005.

[11] M. Vu and A. Paulraj, "MIMO wireless linear precoding", IEEE Signal Processing Magazine, Sep 2007.

[12] S. Venkatesan, A. Lozano and R. Valenzuela, "Network MIMO: Overcoming Intercell Interference in Indoor Wireless Systems", Proceedings of Asilomar 2007.

[13] Z. Jing, D. N. C. Tse, J. B. Soriaga, J. Hou, J. E. Smeet and R. Padovani, "Downlink Macro-Diversity in Cellular Networks", Proceedings of ISIT 2007.

[14] O. Somekh, B. M. Zaidel and S. Shamai, "Sum Rate Characterization of Joint Multiple Cell-Site Processing", IEEE Trans. on Information Theory, Vol. 53, 2007.

[15] D. Gesbert, S. Hanly, H. Huang, S. Shamai (Shitz), O. Simeone, and W. Yu, Multi-cell MIMO cooperative networks: A new look at interference, IEEE Journal on Selected Areas in Communications, Vol. 28, 2010.

[16] S. Ramanath, M. Debbah, E. Altman and V. Kumar, "Asymptotic Analysis of Precoded Small Cell Networks", Proceedings of INFOCOM 2010.

[17] E. Bjornson and E. Jorswieck, Optimal resource allocation in coordinated multi-cell systems, Foundations and Trends in Communications and Information Theory, Vol. 9, 2013.

[18] D. Bethanabhotla, O. Bursalioglu, H. Papadopoulos and G. Caire, "Optimal User-Cell Association for Massive MIMO Wireless Networks", arXiv:1407.6731v2 [cs.NI].

[19] S. Wagner, R. Couillet, M. Debbah, and D. T. M. Slock, "Large system analysis of linear precoding in correlated MISO broadcast channels under limited feedback", IEEE Trans. on Information Theory, Vol. 58, 2012.

[20] A. D. Wyner, "Shannon-Theoretic Approach to a Gaussian Cellular Multiple-Access Channel", IEEE Trans. on Information Theory, Vol. 40, 1994. 